\title{
Stable multisoliton pulses in dispersion management with fiber Bragg gratings
}

\author{
J. D. Ania-Castañón, P. García-Fernández, and J. M. Soto-Crespo \\ Instituto de Óptica, Consejo Superior de Investigaciones Clentificas, Madrid, Spain
}

Received October 26, 1999

\begin{abstract}
We have studied the propagation of prechirped Gaussian pulse pairs in a fiber Bragg grating dispersionmanaged system. We discovered that, under quite general conditions, a number of individual pulses evolve to a stable bound multisoliton solution, with fixed values for the phase difference and the distance between adjacent pulses. These stable multisoliton solutions may propagate for long distances without deformation, with the ultimate distance limitation imposed by the noise amplification. (c) 2000 Optical Society of America OCIS codes: $190.5530,320.1590,270.5530,230.1480$.
\end{abstract}

Dispersion-managed soliton systems,${ }^{1-5}$ in which the dispersion map is controlled by periodic dispersion compensation, may improve the transmission capacity of standard fiber links. Dispersion-managed solitons may exist even when the average network dispersion is zero or positive. ${ }^{6}$ Operating near zero average dispersion reduces the Gordon-Haus jitter ${ }^{7}$ that otherwise limits the performance of long-distance soliton-based systems. Kumar and Hasegawa ${ }^{8}$ recently introduced the concept of the quasi soliton. The quasi soliton is a chirped soliton with quickly decaying Gaussian tails that undergoes periodic amplification and chirp reconstruction. Kumar and Hasegawa ${ }^{8}$ and Turitsyn and Mezentsev ${ }^{10}$ demonstrated that quasi solitons ${ }^{9}$ with strong confinement and reduced interaction between neighbors can be produced by use of ideal fiber Bragg gratings to periodically reverse the pulse chirp.

The interaction between traditional solitons is ruled by the relationship between their phases. If both solitons are at interaction distance, they will be attracted to each other when they are in phase and repelled when they are in antiphase. If the relationship between phases reaches a stationary value or oscillates closely around it, the formation of a bound state is indicated. Afanasjev and Akhmediev ${ }^{11}$ studied the interaction between solitons in a two-dimensional phase plane that they called the interaction plane. This representation has since been used by other authors to analyze the stability properties and general dynamics of multisoliton solutions in different nonconservative optical systems. ${ }^{12,13}$ Here we show that, for long enough propagation distances, stable multisoliton solutions are found in a fiber Bragg grating dispersion-managed system.

Fiber Bragg gratings are usually modeled in dispersion-management analysis as having full reflectivity and producing a linear time delay. ${ }^{10}$ Typical distances between two gratings in a fiber link are $\sim 100 \mathrm{~km}$, and thus the signal passes through 100 fiber gratings in a $10,000-\mathrm{km}$ propagation. With such a high number of gratings, deviations from an ideal model become important, so we obtain the reflectivity and time-delay profiles from the coupled-mode equations of a linearly chirped fiber Bragg grating, ${ }^{14}$ namely,

$$
\begin{aligned}
& \frac{\mathrm{d} R}{\mathrm{~d} z}+i \delta R=-i \kappa(z) S \exp \left[-i F\left(\frac{z}{l}\right)^{2}\right] \\
& \frac{\mathrm{d} S}{\mathrm{~d} z}-i \delta S=i \kappa(z) R \exp \left[i F\left(\frac{z}{l}\right)^{2}\right]
\end{aligned}
$$

where $R$ and $S$ are, respectively, the progressive and the regressive modes inside the grating, $z$ is the length coordinate, $\kappa(z)$ is the coupling coefficient, which includes the chosen apodization function, $F$ is the grating chirp parameter, $l$ is the grating length, and $\delta$ is the frequency deviation with respect to the Bragg frequency at the center of the grating $(z=0)$. When Eqs. (1) and (2) are solved numerically, the reflectivity is $|S / R|^{2}$ and the time of delay is the first frequency derivative of the phase induced by the fiber grating (i.e., the phase term in $S / R$ ).

We consider propagation in an anomalous dispersion fiber of length $L$, following the nonlinear Schrödinger equation including losses. The signal launched into the fiber link is composed of $n$ Gaussian chirped pulses of power $P$ and temporal width $T_{0}$, separated by $\tau_{0}$ :

$U(0, T)$

$$
=P^{1 / 2} \sum_{k=0}^{n-1} \exp \left\{\left[-\frac{(1+i C)\left(T+k \tau_{0}\right)^{2}}{2 T_{0}^{2}}\right]+i \Phi_{k}\right\}
$$

where $C$ represents the initial signal chirp and $\Phi_{k}$ is a constant term added to the phase of each pulse.

Our system is built from dispersion-shifted fiber with dispersion parameter $\beta_{2}=-1 \mathrm{ps}^{2} \mathrm{~km}$, a nonlinear coefficient $\Gamma=0.002 \mathrm{~km}^{-1} \mathrm{~mW}^{-1}$, and fiber loss of $0.2 \mathrm{~dB} \mathrm{~km}^{-1}$. Gratings are placed at $100-\mathrm{km}$ intervals, and amplification takes place after each grating filtering. Time delay and reflectivity for a grating 
with $l=4.78 \mathrm{~cm}, \kappa=4.28 \mathrm{~cm}^{-1}$, and $F=300$, which compensates for the second-order dispersion suffered in the $100-\mathrm{km}$ fiber link, are obtained from the numerical solution of Eqs. (1) and (2) with a 12th-order superGaussian as the apodization function and are depicted in Fig. 1.

The propagation of a pair of pulses $(n=2)$ with $P=25 \mathrm{~mW}, T_{0}=7.8 \mathrm{ps}$, and an initial separation $\tau_{0}=$ 27.34 ps, illustrated in Fig. 2, exemplifies the formation of a bound state of two solitons, which occurs independently of the initial phase difference $\left(\Phi_{1}-\Phi_{0}\right)$ between them. The spectral width of the signal is approximately half of the bandwidth of the grating. The pulses initially suffer distortion and oscillate in amplitude, but they eventually converge to a stable solution in which their powers, the separation $(\tau)$, and the phase difference between them oscillate slightly about fixed values (see Fig. 3). The stability of this bound solution is ultimately limited by the instability of the background. The separation between pulses in the stable bisoliton state (in the example, oscillating near $29 \mathrm{ps}$ ) must be considered a limiting factor on channel capacity for long-haul communication systems with similar characteristics to the one being studied. Similar results are obtained for a range of input peak powers of $20-29 \mathrm{~mW}$ and initial separations between the pulses of $2-5$ pulse widths. Bound solutions of three (see Fig. 4) or more solitons are also possible, though convergence is not guaranteed for arbitrary initial phase differences, as it occurs in the two-soliton case and appears at longer distances. It is of interest to note that a steepened Gaussian fits well the shape of the pulses in the multisoliton state. The steepening, whose origin must lie in an overall dependence of the group velocity on the intensity of the signal, is caused by the successive filterings with nonideal fiber gratings.

In a single period, during the propagation through the $100-\mathrm{km}$ fiber link, the signal is affected by dispersion, self-phase modulation, and losses. Because of these effects the signal becomes wider in the spectral domain, pulses attract each other and nearly merge at the end of the link, and the peak power is reduced to $0.16 \mathrm{~mW}$. The signal is then reconstructed at the fiber grating, recovering its initial chirp, and amplified. Linearly chirped gratings present higher-order dispersion terms in their transfer functions, which are responsible for an asymmetry in their phase responses. These effects can be strongly reduced by application of an appropriate apodization function but may become important when a large number of gratings is used. Thus two competing effects simultaneously affect the signal in the grating. The first one is produced by the combined action of these higher-order terms together with the nonlinear effects in the fiber link and tends to asymmetrize the signal. The second is the filtering effect of the grating, which tends to symmetrize the signal. These competing tendencies reach equilibrium after a certain number of periods. Once this point of equilibrium is reached, the shape of the pulses is approximately fixed, in both the spectral and the time domains. In other words, only slight oscillations around a given point of the interaction plane appear.

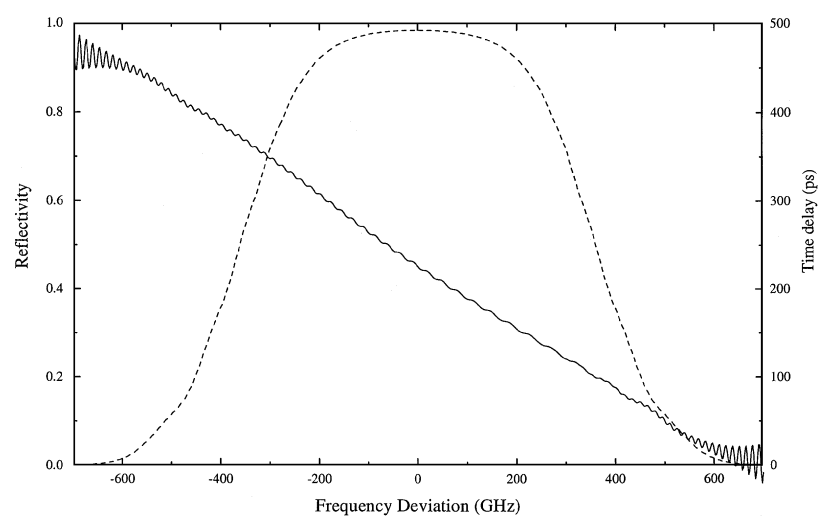

Fig. 1. Reflectivity (dashed curve) and time delay (solid curve) corresponding to any one of the chirped fiber Bragg gratings used for periodic dispersion compensation.

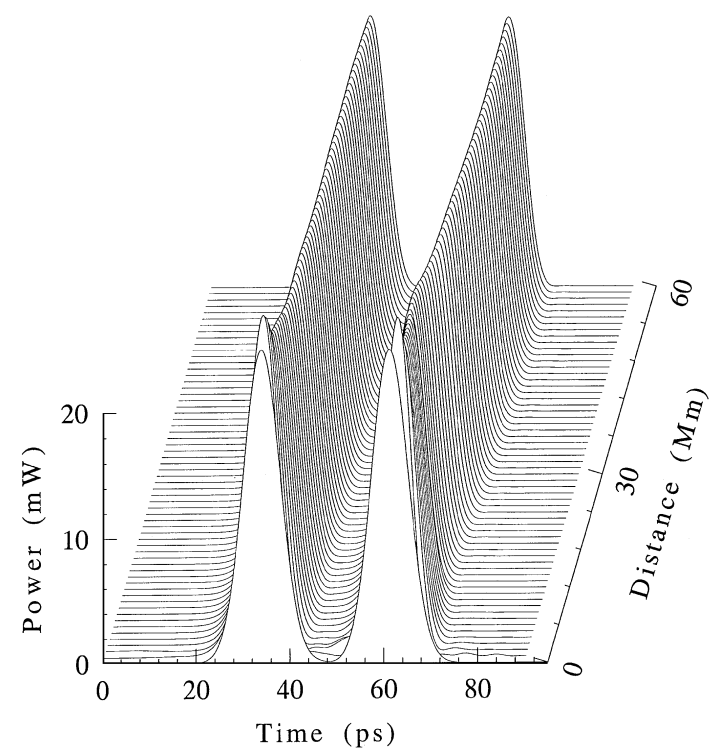

Fig. 2. Evolution of a pulse pair with initial zero phase difference through a distance of $60 \mathrm{Mm}$.

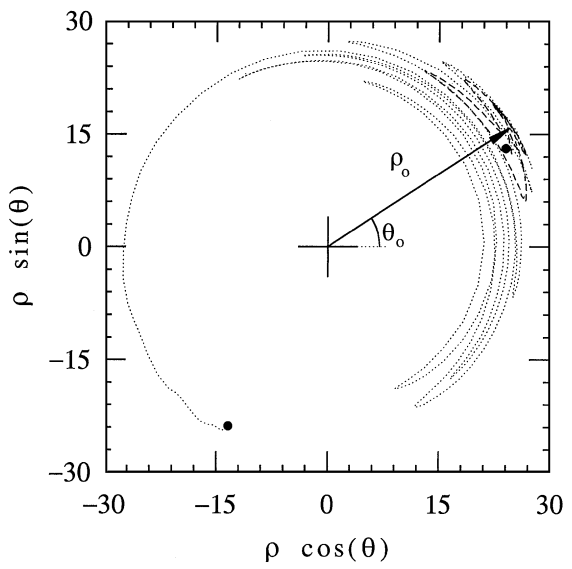

Fig. 3. Trajectories of two pulse pairs with arbitrary initial phase difference in the interaction plane. The initial values are indicated by filled circles. The two trajectories converge to oscillate about the stability point given by $\theta_{o}=0.48 \mathrm{rad}, \rho_{o}=29 \mathrm{ps}$, where $\theta_{o}$ and $\rho_{o}$ are, respectively, the phase difference and the distance between peaks of the bound state. 


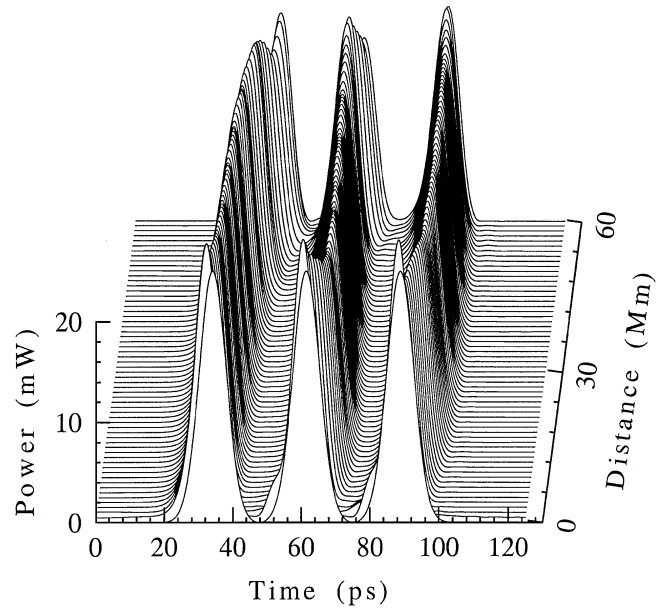

Fig. 4. Evolution of a group of three pulses with an initial phase difference of $\Pi$ between adjacent pulses. Convergence to a stable multisoliton state is slower than in the pulse pair case, but pulse separation is well maintained.

We conclude that multisoliton pulses are naturally generated in nonideal fiber grating dispersionmanaged systems. The inclusion of a full grating model is necessary to explain the effects that lead to the formation of such bound states. The shape of these states, with a fixed distance between peaks, sets a limit on the bit capacity of the channel at distances of several megameters.

J. D. Ania-Castañón thanks the Comunidad de Madrid for financial support. This study has been supported by the Comisión Interministerial de Ciencia y Tecnología, Spain, Project TIC 98-0418-C05-03, Comunidad de Madrid Project 06T-039-96, and the Dirección General de Enseñanza Superior under contract PB-96-0819.

\section{References}

1. N. J. Smith, N. J. Doran, W. Forysiak, and F. M. Knox, J. Lightwave Technol. 15, 1808 (1997).

2. J. P. Gordon and L. F. Mollenauer, Opt. Lett. 24, 223 (1999).

3. D. S. Govan, W. Forysiak, and N. J. Doran, Opt. Lett. 23, 1523 (1998).

4. M. Nakazawa and H. Kubota, Electron. Lett. 31, 216 (1995).

5. C. Paré and P.-A. Bélanger, Opt. Commun. 168, 103 (1999).

6. J. H. B. Nijhof, W. Forysiak, and N. J. Doran, Opt. Lett. 23, 1674 (1998).

7. G. M. Carter, J. M. Jacob, C. R. Menyuk, E. A. Golovchenko, and A. N. Pilipetskii, Opt. Lett. 22, 513 (1997).

8. S. Kumar and A. Hasegawa, Opt. Lett. 22, 372 (1997).

9. Y. Kodama, S. Kumar, and A. Maruta, Opt. Lett. 22, 1689 (1997).

10. S. K. Turitsyn and V. M. Mezentsev, Opt. Lett. 23, 600 (1998).

11. V. V. Afanasjev and N. N. Akhmediev, Phys. Rev. E 53, 6471 (1996).

12. N. N. Akmediev, A. Ankiewicz, and J. M. Soto-Crespo, Phys. Rev. Lett. 79, 4047 (1997).

13. J. M. Soto-Crespo and N. N. Akhmediev, J. Opt. Soc. Am. B 16, 674 (1999).

14. F. Oullette, Opt. Lett. 12, 847 (1987). 